\title{
Reviewers for Volume 22
}

The following colleagues have generously given of their time and energy to review papers for Law and Human Behavior in the last year. We are grateful for their contribution to this journal and the discipline.

Karen Abram
Paul S. Applebaum*
Michael Bagby
Donald N. Bersoff*
Eugene Borgida*
Randy Borum*
Bette Bottoms*
Jack Brigham*
Maggie Bruck
John Carroll
Pamela Casey
Jonathan Casper
Stephen J. Ceci*
Margaret Coggins
Dewey Cornell
Mark Costanzo*
Brian L. Cutler*
Shari Seidman Diamond*
Ronald C. Dillehay
Joel Dvoskin
Donna Eisenstadt
Phoebe C. Ellsworth*
Charles P. Ewing*
David L. Faigman*
Norman J. Finkel*
Louise F. Fitzgerald
Diane R. Follingstad*
Dennis Fox
Patricia A. Frazier
Solomon M. Fulero*
Jeff Gfeller
Judy Gibbons

Karen Abram

Michael Bagby

Randy Borum*

Maggie Bruck

Margaret Coggins

Brian L Cutler*

Shari Seidman Diamond*

Joel Dvoskin

Donna Eisenstadt

Phoebe C. Ellswort

Norman J. Finkel

Louise F. Fitzgerald

Dennis Fox

Patricia A. Frazie

Jeff Gfeller

\author{
James Gilsinan \\ Jonathan M. Golding \\ Stephen L. Golding* \\ Jesse Goldner \\ Gail S. Goodman* \\ Jane Goodman-Delahunty* \\ Edith Greene* \\ J. Thomas Grisso* \\ Barbara A. Gutek* \\ Thomas Hafemeister \\ Paul Handel \\ Craig W. Haney* \\ Valerie P. Hans* \\ Stephen D. Hart \\ Richard Harvey \\ Reid Hastie \\ Jeffrey J. Haugaard* \\ Kirk Heilbrun* \\ Helene Hembrooke \\ Larry Heuer \\ Ronald R. Holden \\ Martin Kaplan \\ Saul Kassin* \\ Norbert L. Kerr \\ Margaret Bull Kovera* \\ Janet Kuebli \\ Richard I. Lanyon* \\ Donna LaVoie \\ Michael Leippe* \\ Murray Levine \\ R. C. L. Lindsay* \\ Marsha B. Liss*
}


Elizabeth Loftus*

James Luginbuhl*

Roy Malpass

Gary B. Melton"

Robert G. Meyer

John Monahan*

Gaxy Moran

Edward P. Mulvey*

Douglas Narby

Michael T. Nietzel*

Lois Oberlander

Maureen $O^{\prime}$ Connor

James $\mathbb{R}$. P. Ogloff

Randy K. Otto*

Paul Paese

Maithilee Pathak

Steven Penrod*

Michael Perlin

John Petrella

Keri Pickel

Norman J. Poythress

John Pryor

Richard Rakos

J. Don Read*

N. Dickon Reppucci*

Marnie E. Rice*

Caton Roberts
Richard Rogers

David F. Ross*

Richard Barry Ruback*

Michael J. Saks

Bruce D. Sales

Karen Saywitz

Regina A. Schuller*

Russell Seawright

John S. Shaw

Christopher Slobogin*

Mark Small

Vicki L. Smith"

Michael E. Sobel

Loretta J. Stalans*

Henry J. Steadman*

Christina A. Studebaker

William C. Thompson

Alan J. Tomkins*

Tom R. Tyler*

Ralph Underwager

Neil Vidmar*

Gary L. Wells*

David B. Wexler*

Audrey T. F. Wiener

Bruce J. Winick*

Roselle L. Wissler

John C. Yuille 